\title{
CONCEPTUALIZACIÓN DE LA GESTIÓN TECNOLÓGICA COMO APORTE A LA INNOVACIÓN ORGANIZACIONAL EN LA EDUCACIÓN SUPERIOR
}

\author{
Alejandro P. Aldás $s^{1,2}$ \\ ${ }^{1}$ Pontificia Universidad Católica del Ecuador Sede Quito \\ ${ }^{2}$ Escuela Politécnica Nacional \\ Autor para correspondencia: aaldas@puce.edu.ec \\ Recibido: 2020/11/02 \\ D0l: https://doi.org/10.26621/ra.v1i24.685 \\ Aprobado: 2021/05/27
}

\section{RESUMEN}

Dentro del contexto histórico, social y cultural de la educación superior en Ecuador, el objetivo de esta investigación documental fue realizar una revisión bibliográfica exploratoria como base para comprender el significado que tiene la tecnología para la comunidad y cómo aporta a la innovación organizacional en las instituciones de educación superior. Mediante la argumentación de conceptos de la Teoría de la Contingencia Estructural y de la Teoría de la Construcción Social de la Tecnología, se aporta a la discusión para encontrar nuevas formas de imaginar la educación superior ecuatoriana, apoyados en la gestión de la tecnología, para dar cabida a estructuras más orgánicas donde prime la racionalidad sustantiva, que promuevan el bienestar, la creatividad y la excelencia de los miembros de la comunidad universitaria. De tal manera, se hará posible que la sociedad reciba personas que busquen la justicia, la equidad social y la emancipación del ser humano. En conclusión, para generar una perspectiva desde el sur que innove la educación superior en Ecuador en el siglo XXI, es necesario considerar el contexto social donde se producen las interacciones entre la tecnología, la cultura en la educación superior y la estructura organizacional de las universidades. Ello se logrará indagando en la conciencia y la subjetividad humanas desde el punto de vista de los miembros de la comunidad universitaria, con el fin de meditar sobre la realidad de la educación superior como una red de significados intersubjetivamente compartidos, que determinan la forma como cada institución de educación superior reacciona a los retos del entorno.

Palabras clave: Universidad, tecnología, administración, pensamiento organizacional, contingencia estructural, construcción social.

\section{ABSTRACT}

Within the historical, social, and cultural context of higher education in Ecuador, the objective of this documentary research was to carry out a bibliographic review as a basis for understanding the meaning that technology has for the community and how it contributes to organizational innovation in higher education institutions. Through the argumentation of concepts of the Structural Contingency Theory and the Theory of the Social Construction of Technology, it contributes to the discussion to find new ways of imagining Ecuadorian higher education, supported by the management of technology, to give room for more organic structures where substantive rationality prevails, which promote the well-being, creativity, and excellence of the members of the university community. In such a way, that society receives people who seek justice, social equity, and the emancipation of the human being. In conclusion, to generate a perspective from the south that innovates higher education in Ecuador in the 21st century, it is necessary to consider the social context where the interactions between technology, culture in higher education, and the organizational structure of universities take place. Inquiring into human consciousness and subjectivity from the point of view of the members of the education community, to meditate on the reality of higher education as a network of intersubjective shared meanings, which determine the way each higher education institution reacts to the challenges of the environment.

Keywords: University, technology, administration, organizational thinking, structural contingency, social construction.

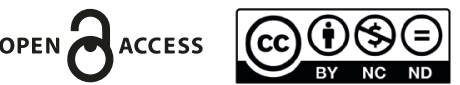




\section{INTRODUCCIÓN}

La recesión económica del Ecuador en los años recientes ha afectado la regularidad de las asignaciones económicas del Estado a las instituciones de educación superior (IES); además, las nuevas modalidades de educación sin fronteras e instituciones educativas con nuevos modelos de costo - calidad, cobertura y modalidades pedagógicas (PUCE, 2016) exige a las IES ecuatorianas poner especial atención en innovar y mejorar significativamente sus niveles de calidad, excelencia académica y eficiencia, para incrementar sus asignaciones presupuestarias, asignar becas a más estudiantes de escasos recursos, brindar una educación superior equitativa y justa a la sociedad, entre otras cuestiones, que les permita afrontar los retos del entorno que exigen cambios y mejoras significativas en la educación superior.

Además, el contexto histórico, social y cultural ecuatoriano obliga continuamente a las IES a abandonar sus comodidades e innovar con el fin de afrontar desafíos como: la internacionalización de los estudios universitarios, la virtualización de la educación superior, la relación con el mundo del trabajo, la calidad de las instituciones educativas, y la gobernanza (Ponce, 2018).

Las tecnologías de la información y la comunicación (TIC) han sido adoptadas por las IES, debido a que son herramientas necesarias en todos los procesos de gestión, investigación, docencia, difusión del arte y la ciencia; permiten operar grandes cantidades de información y bases de datos, y pueden convertirse en eficaces herramientas que soporten la innovación en las universidades (Martínez et al., 2015). Asimismo, Maldonado (2007) señala que las TIC pueden impulsar cambios hacia nuevos modelos educativos más personalizados y centrados en las actividades de los estudiantes, y apoyar las posibilidades de innovación para lograr una educación de mejor calidad y más inclusiva.

Por lo tanto, el objetivo de esta investigación documental es realizar una revisión bibliográfica como base para comprender, con visión crítica y basado en el paradigma interpretativo, el significado que tiene la obtención, adaptación y explotación de la tecnología y cómo influye en la innovación organizacional de las IES para brindar una educación superior de calidad, el bienestar de la comunidad universitaria y afrontar los retos del entorno.

El paradigma interpretativo tiene un enfoque subjetivista del análisis del mundo social (nominalista, antipositivista, voluntarista e ideográfico) y un enfoque en la sociología de la regulación (status quo, orden social, consenso, integración social, cohesión, solidaridad); se orienta a comprender el mundo social subjetivamente creado "tal como es" (Burrell \& Morgan, 2019).

\section{MÉTODOS}

El método utilizado en esta investigación fue una revisión narrativa de la literatura, que permite examinar cualitativamente la bibliografía publicada anteriormente sobre un ámbito de estudio específico, analizarla y discutirla de manera crítica bajo determinadas perspectivas, donde se muestren las coincidencias y contraposiciones del tema abordado, se identifiquen aspectos pendientes y líneas para investigaciones futuras (Pautasso, 2013).

En esta revisión narrativa de la literatura se analizaron y discutieron con visión crítica y con base en el criterio del investigador, artículos científicos y libros relevantes a la problemática abordada, relacionados con el pen- samiento organizacional, la gestión tecnológica, el entorno socioambiental, las tecnologías de la información y la comunicación, la innovación, y los aspectos congruentes con el bien común de la sociedad; asimismo, se argumentaron conceptos de la Teoría de la Contingencia Estructural y de la Teoría de la Construcción Social de la Tecnología, como aporte a la discusión para encontrar nuevas formas de estructuras organizacionales en la educación superior ecuatoriana, apoyados por la gestión de la tecnología.

\section{RESULTADOS Y DISCUSIÓN}

A continuación, se provee un análisis y discusión teórica de la literatura relacionada, que, mediante comparaciones y contrastes, pretende generar un marco conceptual para la discusión de la problemática y la determinación de las conclusiones respectivas.

\section{Pensamiento organizacional}

En primer lugar, abordaremos el concepto de "Organización", que es el objeto de estudio de la administración y puede ser comprendida como "un sistema social complejo, conformado por individuos que interactúan de formas particulares y dinámicas para el logro de determinados objetivos" (Hernández et al., 2007). A su vez, Donaldson (2012) añade que el conjunto recurrente de relaciones entre los miembros de la organización se puede considerar como la estructura social de la organización, y abarca tanto la organización formal prescrita oficialmente como la organización informal.

El problema de investigación se articula con el pensamiento organizacional al considerar a la organización desde la teoría de la contingencia estructural. De acuerdo con Dávila (2001), la teoría de la contingencia sostiene que la estructura organizacional depende o es contingente con respecto a factores como el medio ambiente, el tamaño, la estrategia, la tarea, o la tecnología, por lo que no existe una mejor estructura organizacional apropiada para todas las circunstancias. La base del enfoque contingencial ha sido el estudio comparativo de organizaciones en búsqueda de factores que expliquen las diferencias entre las mismas para identificar las contingencias que deben tomarse en cuenta en el diseño de la estructura organizacional.

Cuando una organización enfrenta un entorno ambiental estable, la estructura organizacional mecanicista es más eficiente debido al uso de procedimientos estándar para desempeñar actividades rutinarias, pero cuando la organización enfrenta un entorno de cambio la flexibilidad de la estructura organizativa orgánica es mejor ya que soporta la necesaria innovación y adaptación (Burns \& Stalker, 2011). En la tabla 1 se presentan las características principales de las estructuras mecanicista y orgánica.

Asimismo, Hage (1965) mostró que las organizaciones centralizadas y formalizadas producían altas tasas de eficiencia pero baja innovación, mientras que las organizaciones descentralizadas y menos formalizadas producían baja eficiencia pero altas tasas de innovación; por lo tanto, si la eficiencia es el objetivo de la organización se requiere una estructura mecanicista, pero si el objetivo es la innovación la estructura requerida es orgánica.

Las organizaciones comúnmente combinan las estructuras mecanicistas y orgánicas; esto se observa al bajar el nivel del análisis a departamentos. Por ejemplo, en los departamentos administrativos de las universidades se tiene una estructura mecanicista, mientras que en los centros de investigación de las facultades se debería tener una estructura or- 
gánica. Asimismo, al bajar al nivel de tareas de enseñanza, se tienen actividades mecanicistas como tomar exámenes o pasar calificaciones, y también se tienen actividades orgánicas como facilitar el aprendizaje grupal, contestar las preguntas de los estudiantes o despertar la curiosidad del estudiante.

Tabla 1. Características de las Estructuras Mecanicista y Orgánica

\begin{tabular}{ll}
$\begin{array}{l}\text { Estructura Mecanicista (previsi- } \\
\text { bilidad) }\end{array}$ & $\begin{array}{l}\text { Estructura Orgánica (adaptabi- } \\
\text { lidad) }\end{array}$ \\
\hline
\end{tabular}

Alta diferenciación horizontal y Alta integración vertical

\begin{tabular}{ll}
\hline Alta formalización & Baja formalización \\
\hline Centralización & Descentralización \\
\hline Estandarización mediante reglas & Ajuste mutuo y redefinición de \\
escritas & tareas \\
\hline Supervisión cercana & Pericia personal y creatividad sin \\
& supervisión \\
\hline
\end{tabular}

Comunicación vertical en forma Comunicación lateral frecuente de instrucciones

\begin{tabular}{ll}
$\begin{array}{l}\text { Adecuado para la eficiencia de la } \\
\text { producción }\end{array}$ & $\begin{array}{l}\text { Adecuado para la creatividad y la } \\
\text { innovación }\end{array}$ \\
\hline
\end{tabular}

Fuente: Adaptado de Hatch \& Cunliffe (2013)

También, Weick (2017) propone mirar la estructura organizacional como un proceso emergente y en desarrollo de rutinas e improvisaciones interactuantes: las improvisaciones organizativas pueden ofrecer nuevas opciones y ayudar a la organización a reaccionar ante una amenaza 0 aprovechar una oportunidad.

Por otro lado, en la perspectiva simbólico-interpretativa, el pensamiento organizacional considera a la organización como socialmente construida. El mundo social es construido por las interpretaciones de objetos, palabras, acciones y eventos, los cuales son comunicados mediante símbolos (Berger \& Luckmann, 1991).

Adicionalmente, los teóricos simbólico interpretativos argumentan desde su ontología subjetivista que una organización es socialmente construida, no existe independientemente de la conciencia humana y la interacción social; afirman que las realidades organizativas surgen a medida que las personas trabajan e interactúan entre sí y con los recursos materiales que les rodean (Hatch \& Cunliffe, 2013). Los teóricos simbólico-interpretativos ven las estructuras organizacionales como creaciones humanas: son obras dinámicas en progreso que surgen de la interacción social y la construcción de significado colectivo.

\section{Gestión tecnológica}

En esta sección abordaremos el concepto de tecnología, pero para esto es importante comprender el concepto de técnica. Ortega y Gasset (1982) indica que la técnica es la transformación que el hombre aplica a la naturaleza para la satisfacción de sus necesidades. La técnica es la adaptación del medio al sujeto. De manera similar, Vieira (2013) señala que la técnica sirve a la vida, produce materialmente los bienes que el hombre necesita, dentro de un sistema de relaciones sociales definidas. en general las formas de producir las cosas (Vieira, 2013). A su vez, Jarvie (2004) indica que la tecnología es "saber cómo" (know how) hacer las cosas en un entorno específico.

La tecnología es el conjunto de reglas de elección racional que determinan las formas de proceder sobre los medios técnicos, para una eficaz realización de fines. Los medios técnicos pueden ser instrumentos, máquinas y autómatas que posibilitan un ahorro del trabajo (Habermas et al., 2000).

Una vez revisado el concepto de tecnología, es momento de abordar el concepto de "gestión tecnológica", la cual comprende las actividades vinculadas a la identificación, adquisición, desarrollo, adaptación y protección de tecnologías que permitan incrementar la capacidad de las organizaciones, con el fin de alcanzar sus objetivos (Solleiro \& Castañon, 2016).

Asimismo, la gestión tecnológica abarca la conducción de los procesos relacionados con la tecnología, maneja los cambios tecnológicos en las organizaciones, y tiene dos enfoques principales: la innovación y el mejoramiento continuo (Castellanos-Domínguez, 2003). Por otra parte, Orr (2016) indica que la gestión de la tecnología no se trata solo de la tecnología en sí misma, sino que también incluye la administración de las interacciones realizadas por las personas que usan la tecnología.

En la teoría de la contingencia estructural, la tecnología es considerada como un factor de contingencia, que tiene su origen en las investigaciones de Joan Woodward, quien agrupó las organizaciones de acuerdo a su nivel de complejidad tecnológica, y encontró que las organizaciones tenían un mejor desempeño (George et al., 2005), en situaciones donde:

- las tecnologías de producción en masa eran combinadas con estructuras mecanicistas con alta rutina de trabajo, y

- las tecnologías de lotes pequeños eran combinadas con estructuras orgánicas con baja rutina de trabajo

Por ejemplo, en las aulas de las universidades tenemos tecnologías de lotes pequeños por lo que la estructura orgánica sería la adecuada para un buen desempeño.

Por otra parte, Thompson (2017) propone una tipología tecnológica basada en distinguir entre tres tecnologías:

- la primera Intensiva (con coordinación de habilidades especializadas como en un hospital),

- la segunda Mediadora (que conectan clientes uniéndolos en un intercambio), y

- la tercera de Enlace largo (con procesos de transformación lineal como en líneas de ensamblaje).

Por ejemplo, las facultades en las universidades pueden tener una tecnología mediadora ya que conectan una variedad de estudiantes y profesores en las aulas de clase.

Por su lado, Perrow (1967) para desarrollar su tipología tecnológica (Tabla 2) definió la Variabilidad de tareas (cantidad de excepciones a los procedimientos estándar) y el Análisis de las tareas (métodos conocidos para manejar la excepción) como medio para diferenciar las tecnologías. Por ejemplo, en los centros de investigación se deben tener tecnologías no rutinarias debido a las tareas creativas que llevan a cabo. 
Tabla 2. Tipología tecnológica de Perrow

Variabilidad de la tarea

\begin{tabular}{llll}
\cline { 2 - 3 } \multicolumn{2}{c}{ Baja } & Alta \\
\hline $\begin{array}{l}\text { Análisis } \\
\text { de la }\end{array}$ & Alta & $\begin{array}{l}\text { Rutina (producción } \\
\text { en masa) }\end{array}$ & Ingeniería (diseño) \\
\cline { 2 - 4 } tarea & Baja & Artesanía (costura) & No rutina (investigación) \\
\hline
\end{tabular}

Fuente: adaptado de (Hatch \& Cunliffe, 2013)

De manera similar, existen proposiciones sobre los efectos de la tecnología en la estructura organizacional, por ejemplo, un efecto de la tecnología en la formalización es descrito mediante la siguiente proposición: si la rutina tecnológica es baja, entonces la formalización debería ser baja (Burton \& Obel, 2004).

Adicionalmente, Chiavenato (2006) añade que todas las organizaciones utilizan tecnología en alguna forma para realizar sus tareas; así, la tecnología adoptada puede ser rudimentaria o sofisticada. Sin embargo, todas las organizaciones dependen de un tipo de tecnología para poder funcionar y alcanzar sus metas. Por ejemplo, se puede describir la tecnología básica de una universidad como la investigación y la educación, cuando bajamos al nivel de actividades:

- en las tecnologías de enseñanza se encuentran las técnicas de participación en el aula,

- en las tecnologías de investigación se encuentra el diseño de investigación, y

- en las tecnologías administrativas se encuentra la matriculación de los estudiantes.

En contraste, la teoría de la construcción social de la tecnología visibiliza cómo las preocupaciones no técnicas (como las normas culturales y las expectativas sociales) moldean la tecnología. En la perspectiva simbólico-interpretativa, los teóricos de la organización creen que la tecnología es socialmente construida y, por lo tanto, la tecnología no solo se refiere a objetos físicos, sino también a símbolos que incluyen palabras, imágenes y metáforas; no solo se centran en las actividades de tareas rutinarias, sino también en las interacciones entre las personas y la tecnología, de manera que la interpretación de significados se vuelve tan importante como el conocimiento de la tecnología (Hatch \& Cunliffe, 2013).

También, Orlikowski (2008) indica que la estructura organizacional surge tanto de las propiedades físicas de la tecnología, como de las formas en que las personas construimos e interactuamos con dicha tecnología. Las tecnologías son formadas por complejas compensaciones socioculturales, y proponen un modelo evolutivo de innovación tecnológica que expone el papel de la construcción social en el desarrollo de la tecnología (Pinch \& Bijker, 2012).

Del mismo modo, el estudio etnográfico de Orr (2016) sobre el trabajo de los técnicos de reparación de fotocopiadoras de Xerox, concluyó que las fotocopiadoras tienen una presencia tanto técnica como social: las máquinas individuales también tienen sus propias historias y formas de comportarse. El estudio de Orr no solo resaltó la naturaleza socialmente construida y situada de la tecnología, sino que confirmó que los conceptos de tecnología, estructura organizacional y cultura en la organización se influyen mutuamente.

A propósito de la cultura en la organización, Aguirre (2002) señala que es un conjunto de elementos interactivos (creencias, valores, comportamientos) generados y compartidos por los miembros de una organización al tratar de conseguir sus objetivos, que cohesionan e identifican.

\section{Entorno socioambiental}

En este apartado, abordaremos el concepto de entorno socioambiental, que en la teoría de la contingencia estructural se define como una entidad objetiva que se encuentra fuera de los límites de la organización (Hatch \& Cunliffe, 2013).

Decidir qué elementos están dentro o fuera de los límites organizacionales puede ser difícil y depende del propósito del análisis. Por ejemplo, en una universidad que quiere examinar los efectos de subir los costos de matrícula puede ser útil considerar a los estudiantes como clientes, es decir miembros del entorno; pero si la universidad quiere solicitar fondos de investigación externos, entonces sería útil considerar a los estudiantes como miembros de la organización que se beneficiarán de las actividades de investigación.

En este contexto, las características organizacionales reflejan la influencia del entorno socioambiental en el que se encuentra la organización; por lo tanto, para que la organización sea eficaz, debe ajustar su estructura a los factores de contingencia de la organización y por consiguiente al medio ambiente (Donaldson, 2012).

Por su parte, Lawrence y Lorsch (1967) señalan que la incertidumbre del entorno socioambiental (definida como la interacción entre la complejidad de elementos del ambiente y la velocidad del cambio ambiental) afecta la diferenciación e integración en las estructuras organizacionales.

Por el contrario, desde la perspectiva simbólico-interpretativa, el entorno socioambiental se define como una construcción social que surge de la representación, el mapeo cognitivo y los procesos de creación de sentido; el entorno surge del simbolismo y las creencias intersubjetivamente compartidas sobre el medio ambiente. Las diferentes organizaciones construyen sus entornos de manera diferente, y la misma organización cambiará su comportamiento en respuesta a su entorno socioambiental cuando su construcción cambie (Hatch \& Cunliffe, 2013). Por ello, los construccionistas sociales ven la tecnología como el producto de la interacción de factores sociales, culturales y económicos en el entorno socioambiental.

\section{Tecnologías de la información y la comunicación}

En esta sección, abordaremos el concepto de las Tecnologías de la Información y la Comunicación (TIC) que, de acuerdo con Cobo (2009), se definen como:

Dispositivos tecnológicos (hardware y software) que permiten producir, editar, almacenar, intercambiar y transmitir datos entre diferentes sistemas de información que cuentan con protocolos comunes. Estas aplicaciones, que integran medios de informática, telecomunicaciones y redes, posibilitan tanto la comunicación y colaboración interpersonal (persona a persona) como la multidireccional (uno a muchos o muchos a muchos). Estas herramientas desempeñan un papel sustantivo en la generación, intercambio, difusión, gestión y acceso al conocimiento.

Las TIC son un medio para que una organización procese información; incluso la organización misma es una entidad procesadora de información. Entonces, las TIC son un medio para que la institución cumpla con su tarea fundamental. El diseño organizacional ayuda a organizar la forma como la información es procesada, por lo que diferentes tecnologías de la información son requeridas para diferentes diseños organizacionales (Burton \& Obel, 2004)

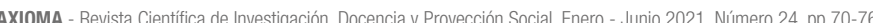
ISSN: 1390-6267- E-ISSN: 2550-6684 


\section{Innovación}

Una innovación es la introducción de nuevas combinaciones de procedimientos u objetos, que siendo nuevos o no, son utilizados de manera original en un contexto dado (Schumpeter \& Backhaus, 2003). Por otro lado, una innovación es un cambio que requiere un considerable grado de imaginación y constituye una rotura relativamente profunda con la forma establecida de hacer las cosas y con ello crea fundamentalmente nueva capacidad (Nelson \& Winter, 2002).

De acuerdo al Manual de Oslo, una innovación organizacional es "la introducción de un nuevo método organizativo en las prácticas, la organización del lugar de trabajo o las relaciones exteriores" (OECD \& Eurostat, 2007)

Además, Burns y Stalker (2011) encontraron que la innovación tiende a ser limitada en las estructuras mecanicistas ya que impiden la flexibilidad y la creatividad necesarias para adaptarse y responder a los cambios ambientales, mientras que las estructuras orgánicas favorecen la innovación ya que brindan mayor poder de decisión a los empleados que no están sujetos a la formalidad de reglas y procedimientos.

Las organizaciones, para desarrollar procesos de innovación deben tener una estructura organizacional receptiva al cambio que les permita formar parte de redes interorganizacionales, y disponer de formas de gestionar el conocimiento de manera eficiente (Tushman \& Nelson, 1990). De este modo, la interacción en las redes interorganizacionales genera procesos de aprendizaje y difusión tecnológica que permite la circulación de conocimientos, creando efectos positivos de retroalimentación que harán más factibles los procesos de innovación (Formichella, 2005).

Entonces, la gestión adecuada de la tecnología puede facilitar la creación y el intercambio de conocimientos; este proceso crea una base de conocimiento que puede ser explotada en la generación de ideas (Scuotto et al., 2017). Por lo tanto, la generación de una innovación involucra tanto la capacidad de aprendizaje organizacional como la capacidad de generar redes heterogéneas de actores; esto permite, a través de procesos colectivos, convertir una idea en una innovación (Daza, 2015).

Por su parte, Morgan (1999) propuso el método de la Imaginización, para llevar a cabo procesos de innovación organizacional. Este método potencia la capacidad de comprender las situaciones organizacionales de nuevas maneras y ayuda a comprender la verdadera naturaleza de los problemas existentes. La Imaginización provee una herramienta poderosa para derribar las barreras del pensamiento burocrático e impulsar los sistemas autoorganizados que se adapten constantemente a los cambios del entorno y utiliza el poder de la imaginación y las analogías para generar respuestas innovadoras a los problemas. Por lo tanto, la Imaginización invita a la creatividad y pretende desarrollar la aptitud para encontrar imágenes o metáforas que permitan leer las situaciones organizacionales desde nuevas perspectivas y nos ayuden a encontrar nuevas maneras de organizar.

\section{Bien común de la sociedad}

La Constitución de la República del Ecuador (2008) en su art. 352 determina que el Sistema de Educación Superior "estará integrado por universidades y escuelas politécnicas; [...] debidamente acreditados y evaluados. Estas instituciones, sean públicas o particulares, no tendrán fines de lucro". Por su parte, la Ley Orgánica de Educación Superior del Ecuador (LOES) (2018) en su art. 159 indica que las instituciones de educación superior son "comunidades académicas con personería jurídica propia, esencialmente pluralistas y abiertas a todas las corrientes y formas del pensamiento universal expuestas de manera científica. Gozarán de autonomía académica, administrativa, financiera y orgánica, [...]”.

Además, la Ley Orgánica de Educación Superior (LOES) (2018) en su art. 160 menciona que corresponde a las universidades y escuelas politécnicas:

Producir propuestas y planteamientos para buscar la solución de los problemas del país; propiciar el diálogo entre las culturas nacionales y de éstas con la cultura universal; la difusión y el fortalecimiento de sus valores en la sociedad ecuatoriana; la formación profesional, técnica y científica de sus estudiantes, profesores e investigadores, contribuyendo al logro de una sociedad más justa, equitativa y solidaria, en colaboración con los organismos del Estado y la sociedad.

En el contexto de la misión encomendada en la LOES a las universidades, los aspectos congruentes con el bien común de la sociedad son la búsqueda constante de nuevas formas de imaginar la estructura organizacional de las IES, apoyados en la gestión de la tecnología, para dar cabida a estructuras más orgánicas donde prime la racionalidad sustantiva, que promuevan el bienestar, la creatividad y la excelencia de los miembros de la comunidad universitaria, de tal manera que la sociedad reciba hombres y mujeres parentéticos que busquen la justicia, la equidad social y la emancipación del ser humano.

El bienestar es la vida que el hombre inventa cuando ya ha superado sus necesidades básicas y tiene tiempo para dedicarse a actividades no biológicas como imaginar, pensar y razonar. Por ello, el bienestar y no el simplemente estar, es la necesidad fundamental para el hombre, ya que determina el estar bien, el vivir bien, el estar satisfecho consigo mismo (Ortega y Gasset, 1982). Por su parte, Guerreiro-Ramos (1981) indica que las características del hombre parentético son su capacidad para la crítica, su necesidad de contar con un propósito en la vida, su aspiración de libertad y su compromiso con la ética.

La organización debe ser un sitio para la emancipación del ser humano a través del trabajo, donde la racionalidad sustantiva provea la esperanza de que los elementos constitutivos de las organizaciones, estén estos basados en la autorrealización, el entendimiento, el juicio ético, la autenticidad, los valores emancipatorios o la autonomía (Serva, 1997). De forma similar, Kolm (2006) plantea que la reciprocidad es el principio orientador de las organizaciones en donde prima la racionalidad sustantiva; implica el compartir y el alegre convivir, el no pensar solo en el lucro.

Finalmente, Tenório (2016) nos enseña que mediante los conocimientos de la razón funcional no lograremos la comprensión clara de las relaciones sociales que se producen dentro de la organización, debido a que la razón funcional ha fragmentado el conocimiento organizacional; el lenguaje de la contradicción servirá para contrarrestar el aletargamiento de la teoría administrativa. Por lo tanto, se necesitan nuevos conceptos que nos permitan reflexionar sobre la racionalidad administrativa, revolucionar las teorías organizacionales y alcanzar la emancipación del ser humano.

\section{CONCLUSIONES}

Gestionar la tecnología para potenciar procesos de innovación organizacional en las universidades puede catalizar la ruptura de las estructuras mecanicistas y derribar las barreras del pensamiento funcionalista, e impulsar la adopción de estructuras orgánicas que promuevan 
la creatividad, la flexibilidad y la adaptabilidad en las actividades académicas y administrativas.

Para impulsar procesos de innovación en la estructura organizacional de Ias IES es necesario no solamente gestionar las tecnologías adecuadas, sino también comprender las interacciones e interpretaciones de significados que hacen los usuarios de dichas tecnologías, para posibilitar a las IES generar respuestas apropiadas a los retos del entorno, fomentar la investigación y educar a los estudiantes en lugar de adiestrarlos.

En el entorno de las IES ecuatorianas, es relevante estudiar la forma cómo se pueden identificar, adquirir, desarrollar y adaptar las tecnologías que promuevan la capacidad innovadora de las IES, de manera que permitan estimular procesos de transformación de la sociedad y persigan la emancipación del ser humano.

La naturaleza socialmente construida y situada de la tecnología implica que la misma es a la vez causa y consecuencia de la estructura organizacional, que la estructura organizacional condiciona y es a la vez un producto de la interacción humana, y que la tecnología es creada por la interacción humana y a su vez la tecnología determina la interacción humana.

El diálogo entre las perspectivas modernista-funcionalista y simbólico-interpretativo de la teoría organizacional permitirá desarrollar una visión más exhaustiva de las relaciones entre la tecnología y la estructura organizacional, que hagan emerger nuevas formas organizacionales mediante las cuales se propicie el bienestar de la comunidad.

\section{Agradecimientos}

Agradezco a la Pontificia Universidad Católica del Ecuador y a la Escuela Politécnica Nacional por el apoyo recibido para la realización de esta investigación. Agradezco en especial al Dr. Efraín Naranjo, por su guía y consejos para la consecución de esta investigación.

Contribución de los autores: El autor ha participado en la redacción del trabajo y análisis de los documentos.

Fuente de financiamiento: Esta investigación fue financiada por la Pontificia Universidad Católica del Ecuador Sede Quito.

Conflicto de intereses: El autor declara no tener conflictos de intereses.

\section{REFERENCIAS}

Aguirre Baztán, S. Á. (2002). La cultura de la empresa. Revista Mal-estar E Subjetividade, 2(2), 86-122. https://www.redalyc.org/articulo. oa? id $=27120205$

Asamblea Constituyente de Montecristi. (2008). Constitución del Ecuador de 2008. Registro Oficial, Órgano del Gobierno del Ecuador. Ciudad Alfaro, 449, 218 p. https://biblioteca.defensoria.gob. ec/bitstream/37000/2726/1/Constitución de la República del Ecuador.pdf

Asamblea Nacional del Ecuador. (2018). Ley Orgánica Reformatoria a Ley Orgánica De Educación Superior. https://www.ces.gob.ec/ documentos/Normativa/LOES.pdf

Berger, P. L., \& Luckmann, T. (1991). A Treatise in the Sociology of Knowledge. The Social Construction of Reality. http://perflensburg.se/Berger social-construction-of-reality.pdf
Burns, T., \& Stalker, G. M. (2011). The Management of Innovation. Oxford University Press. https://doi.org/10.1093/acprof:0so/9780198288787.001.0001

Burrell, G., \& Morgan, G. (2019). Sociological Paradigms and Organisational Analysis. En Sociological Paradigms and Organisational Analysis. https://doi.org/10.4324/9781315609751

Burton, R. M., \& Obel, B. (2004). Technology BT - Strategic Organizational Diagnosis and Design: The Dynamics of Fit (R. M. Burton \& B. Obel (eds.); pp. 237-270). Springer US. https://doi. org/10.1007/978-1-4419-9114-0_7

Castellanos-Domínguez, 0. (2003). Gestión en tecnología: Aproximación conceptual y perspectivas de desarrollo. INNOVAR, revista de ciencias administrativas y sociales, 1(21), 16. http://www.scielo.org.co/pdf/inno/v13n21/v13n21a14.pdf

Chiavenato, I. (2006). Introducción a la Teoría General de la Administración (Séptima). McGraw-Hill. https://esmirnasite.files.wordpress.com/2017/07/i-admon-chiavenato.pdf

Cobo, J. C. (2009). El concepto de tecnologías de la información. Benchmarking sobre las definiciones de las TIC en la sociedad del conocimiento. Zer - Revista de Estudios de Comunicación, 14, 285-318. http://www.ehu.es/zer/hemeroteca/pdfs/zer27-14cobo.pdf

Dávila, C. (2001). Teorías Organizacionales y Administración: Enfoque Crítico. En McGraw-Hill. https://repositorio.uniandes.edu.co/ handle/1992/46537

Daza, C. (2015). Modelo de efectividad de la red de innovación tecnológica. Una visión desde el constructivismo social. [Universidad Andina Simón Bolivar]. https://repositorio.uasb.edu.ec/handle/10644/5038

Donaldson, L. (2012). The Normal Science of Structural Contingency Theory. En Studying Organization: Theory \& Method(2012). SAGE Publications Ltd. https://doi.org/10.4135/9781446218556.n2

Formichella, M. (2005). La Evolución del Concepto de Innovación y su Relación con el Desarrollo. Compras y existencias, 140. https:// core.ac.uk/download/pdf/48031881.pdf

George, J., Claude, S., \& Álvarez, L. (2005). Historia del pensamiento administrativo. En Historia del pensamiento administrativo. https:// es.scribd.com/document/403023233/Historia-Del-Pensamiento-Administrativo-Claude-S-George-y-Alvarez

Guerreiro-Ramos, A. (1981). The New Science of Organizations. A Reconceptualization of the Wealth of Nations. University of Toronto Press. https://utorontopress.com/us/the-new-sciences-organizations-1

Habermas, J., Torres, S., \& Moya, C. (2000). Teoría y praxis. Estudios de filosofía social (Cuarta). Tecnos. https://dialnet.unirioja.es/ servlet/libro?codigo $=326814$

Hage, J. (1965). An Axiomatic Theory of Organizations. Administrative Science Quarterly, 10(3). https://doi.org/10.2307/2391470

Hatch, M., \& Cunliffe, A. (2013). Organization Theory: modern, symbolic and posmodern perspectives (Third). Oxford University Press. http://libsearch.cbs.dk/primo_library/libweb/action/ dIDisplay.do?docld=CBS01000589624\&vid=CBS\&after PDS=true

Hernández, A. A., Saavedra, J., \& Sanabria, J. (2007). Hacia la construcción del objeto de estudio de la administración: Una visión desde la complejidad. Revista Facultad de Ciencias Económicas: Investigación y Reflexión, 15(1), 91-112. https://www.redalyc. org/pdf/909/90915106.pdf

Jarvie, I. C. (2004). La tecnología y la estructura del conocimiento. En C. Mitcham \& R. Mackey (Eds.), Filosofía y tecnología (pp. 49-62). Ediciones Encuentro. www.ediciones-encuentro.es 
Kolm, S. C. (2006). Chapter 6 Reciprocity: Its Scope, Rationales, and Consequences. En Handbook of the Economics of Giving, Altruism and Reciprocity (Vol. 1). https://doi.org/10.1016/S15740714(06)01006-2

Lawrence, P., \& Lorsch, J. (1967). Differentiation and Integration in Complex Organizations. Administrative Science Quarterly, 12(1), 1-47. https://doi.org/10.2307/2391211

Maldonado, L. M. (2007). Las tecnologías de información y comunicación un recurso estratégico para la educación superior. Visión Gerencial, 6(2), 257-268. https://www.redalyc.org/ pdf/4655/465545876011.pdf

Martínez, M., Ruiz, J., Estrada, C., \& Sánchez, M. de la L. (2015). Las tecnologías de la información y comunicación y su impacto en la innovación organizacional en las instituciones de Educación Superior. Revista de Aplicación Científica y Técnica, 1(2), 153-164. http://ri.uaemex.mx/bitstream/handle/20.500.11799/49477/ Tic e innovación.pdf?sequence $=1$ \&isAllowed $=y$

Morgan, G. (1999). IMAGIN-I-ZACIÓN. Ediciones Granica. http://www. granica.com

Nelson, R., \& Winter, S. (2002). Evolutionary Theorizing in Economics. Journal of Economic Perspectives, 16, 23-46. https://doi. org/10.1257/0895330027247

OECD, \& Eurostat. (2007). Manual de Oslo. Guía para la recogida e interpretación de datos sobre innovación. En Tragsa (Vol. 30, Número 5). http://www.oecd-ilibrary.org/science-and-technology/ manual-de-oslo_9789264065659-es

Orlikowski, W. J. (2008). Using Technology and Constituting Structures: A Practice Lens for Studying Technology in Organizations BT Resources, Co-Evolution and Artifacts: Theory in CSCW (M. S. Ackerman, C. A. Halverson, T. Erickson, \& W. A. Kellogg (eds.); pp. 255-305). Springer London. https://doi.org/10.1007/9781-84628-901-9_10

Orr, J. E. (2016). Talking about machines: An ethnography of a modern job. En Talking about Machines: An Ethnography of a Modern Job. https://doi.org/10.2307/3034516

Ortega y Gasset, J. (1982). Meditación de la técnica. Obras de José Ortega y Gasset, 21. https://francescllorens.files.wordpress. com/2013/02/ortega_meditacion_tecnica.pdf

Pautasso, M. (2013). Ten Simple Rules for Writing a Literature Review. PLoS Computational Biology, 9(7). https://doi.org/10.1371/journal.pcbi.1003149

Perrow, C. (1967). A framework for comparative organizational analysis. American Sociological Review, 32(2), 194-208. https://doi.org/ https://doi.org/10.2307/2091811

Pinch, T. J., \& Bijker, W. E. (2012). The social construction of facts and artifacts: Or how the sociology of science and the sociology of technology might benefit each other. En The Social Construction of Technological Systems: New Directions in the Sociology and History of Technology: Anniversary Edition. https://doi.org/ https://doi.org/10.1177/030631284014003004

Ponce, F. (2018). Las universidades católicas ecuatorianas ante la Veritatis Gaudium. Simposio sobre la Constitución Apostólica Veritatis Gaudium, 16. http://conferenciaepiscopal.ec/ponencias-simposio-sobre-la-constitucion-apostolica-veritatis-gaudium/

PUCE. (2016). Plan Estratégico de Desarrollo Institucional 2016-2020. https://www.puce.edu.ec/intranet/documentos/plan-estrategico/PUCE-PEDI-2016-2020-Version-extendida.pdf

Schumpeter, J., \& Backhaus, U. (2003). The Theory of Economic Development BT - Joseph Alois Schumpeter: Entrepreneurship, Style and Vision (J. Backhaus (ed.); pp. 61-116). Springer US. https:// doi.org/10.1007/0-306-48082-4_3
Scuotto, V., Santoro, G., \& Bresciani, S. (2017). Shifting intra- and inter-organizational innovation processes towards digital business: An empirical analysis of SMEs. Creativity and Innovation Management, 26(3), 247-255. https://doi.org/https://doi. org/10.1111/caim.12221

Serva, M. (1997). A racionalidade substantiva demonstrada na prática administrativa. Revista de Administração de Empresas, 37(2). https://doi.org/10.1590/s0034-75901997000200003

Solleiro, J. L., \& Castañon, R. (2016). Gestión tecnológica: conceptos y prácticas (Segunda). https://cambiotec.org.mx/site/wp-content/ uploads/2017/09/E-Libro-Gestión-2.pdf

Tenório, F. G. (2016). Tem razao a administracao?: ensaios de teoria organizacional (4.a ed.). Editora Unijui.

Thompson, J. D. (2017). Organizations in action: Social science bases of administrative theory. En Organizations in Action: Social Science Bases of Administrative Theory. https://doi. org/10.4324/9781315125930

Tushman, M., \& Nelson, R. (1990). Introduction : Technology , Organizations, and Innovation. Administrative Science Quarterly, 35(1), 1-8. https://doi.org/https://doi.org/10.2307/2393548

Vieira, Á. (2013). O conceito de Tecnologia - Volume 1. Contraponto.

Weick, K. E. (2017). Improvisation as a mindset for organizational analysis. En The Aesthetic Turn in Management. https://doi. org/10.4324/9781351147965-19 